\title{
Light meson emission in (anti)proton induced reactions
}

\section{E.A. Kuraev}

JINR-BLTP, 141980 Dubna, Moscow region, Russian Federation

\section{E.S. Kokoulina}

JINR-VBLHE, 141980 Dubna, Moscow region, Russian Federation

\section{E. Tomasi-Gustafsson*}

CEA,IRFU,SPhN, Saclay, 91191 Gif-sur-Yvette Cedex, France and and Univ. Paris-Sud, IPNO, UMR-8608, Orsay, F-91405, France

E-mail: etomasileea.fr

Reactions induced by high energy antiprotons on proton or nuclei are accompanied by the emission of a few mesons with large probability. Interesting phenomena can be observed and QCD tests can be performed, through the detection of one or more mesons. The collinear emission from high energy (anti)proton beams of a hard pion or vector meson, can be calculated similarly to the emission of a hard photon from an electron. This is a well known process in QED, and it is called the "Quasi-Real Electron method", where the incident particle is an electron and a hard photon is emitted leaving an 'almost on shell' electron impinging on the target. In case of emission of a charged light meson, $\pi$ or $\rho$, in proton-proton(anti-proton) collisions, the meson can be deviated in a magnetic field and detected. The collinear emission (along the beam direction) of a charged meson may be used to produce high energy (anti)neutron beams. This can be very useful to measure the difference of the cross sections of (anti)proton and (anti)neutron scattering from the target and may open the way for checking sum rules with antiparticles. Hard meson emission allows also to enhance the cross section when the energy loss from one of the incident particles lowers the total energy up to the mass of a resonance. The cross section is calculated on the basis of factorized formulas, where the probability of emission of the light mesons multiplies the cross section of the sub-process. Multiplicity distributions for neutral and charged meson production are also given.

XXII International Baldin Seminar on High Energy Physics Problems,

15-20 September 2014

JINR, Dubna, Russia

*Speaker. 


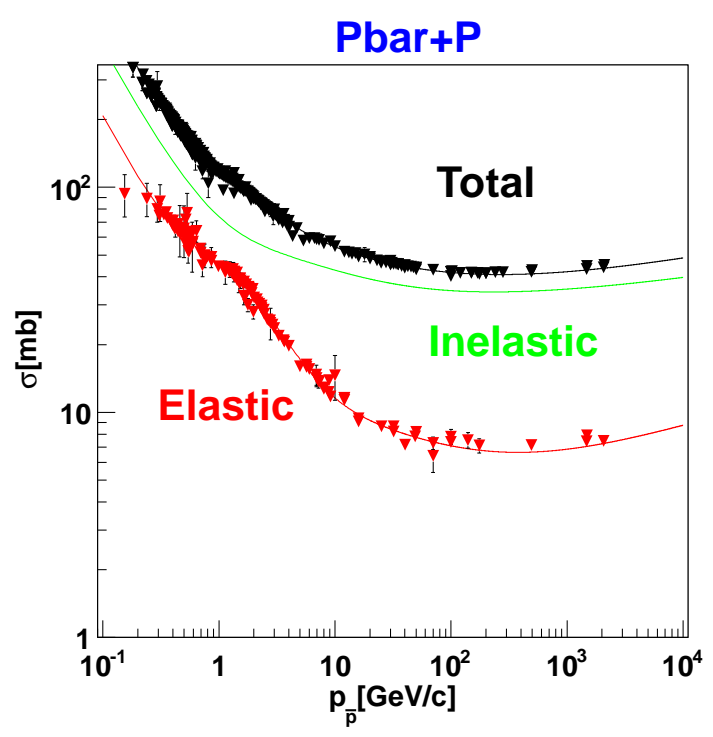

Figure 1: Total (black) and elastic (red) cross section for the $\bar{p}+p$ reaction, as function of the antiproton beam momentum $p_{\bar{p}}$. The contribution of inelastic events is also shown (green line).

\section{Introduction}

Among (anti)proton induced reactions which occur with high probability (the cross section is of the order of [mb]), we focus here to light meson production in the kinematical domain accessible at the future FAIR facility by the PANDA experiment (the antiproton momentum is in the range $1.5-15 \mathrm{GeV})$.

The world data for the total and elastic cross sections for $\bar{p}+p$ induced reactions are illustrated in Fig. 1 as function of the laboratory antiproton momentum [2]. From the figure we see that $\sigma_{\bar{p} p}^{e l} / \sigma_{\bar{p} p}^{t o t} \simeq 1 / 5$ and that the difference between the total and elastic cross section parametrizations (green line), which represents the contribution of the inelastic events, is of the order of $40 \mathrm{mb}$ in the range accessible at PANDA.

Fig. 2 shows that the most probable reaction involving pions, corresponds to more than three pions in the final state. We parametrize the cross section for the reactions given in Fig. 2 as: Among the inelastic processes, the charge exchange process $p \bar{p} \rightarrow n \bar{n}$ has the largest cross section:

It is known from QED that two Feynman diagrams contribute to the process of Bhabhascattering: the scattering $(t)$ and the annihilation $(s)$ channels. Neglecting the interference one can estimate the relative contribution of the scattering diagram $\left|\mathscr{M}_{s}\right|^{2}$ and the annihilation one $\left|\mathscr{M}_{a}\right|^{2}$ for the case when the final particles are emitted at large angles in the system of center of mass:

$$
\frac{\sum\left|\mathscr{M}_{a}\right|^{2}}{\sum\left|\mathscr{M}_{s}\right|^{2}} \approx \frac{1}{10}
$$

The contribution of scattering mechanism dominates. This statement holds even if hadron form factors are taken into account, i.e., one can assume that a similar relative contributions of these two mechanisms would take place in case of elastic and inelastic $\bar{p} p \rightarrow \bar{p} p$ cross section. The processes relevant to "scattering" mechanism are denoted as peripheral processes. 

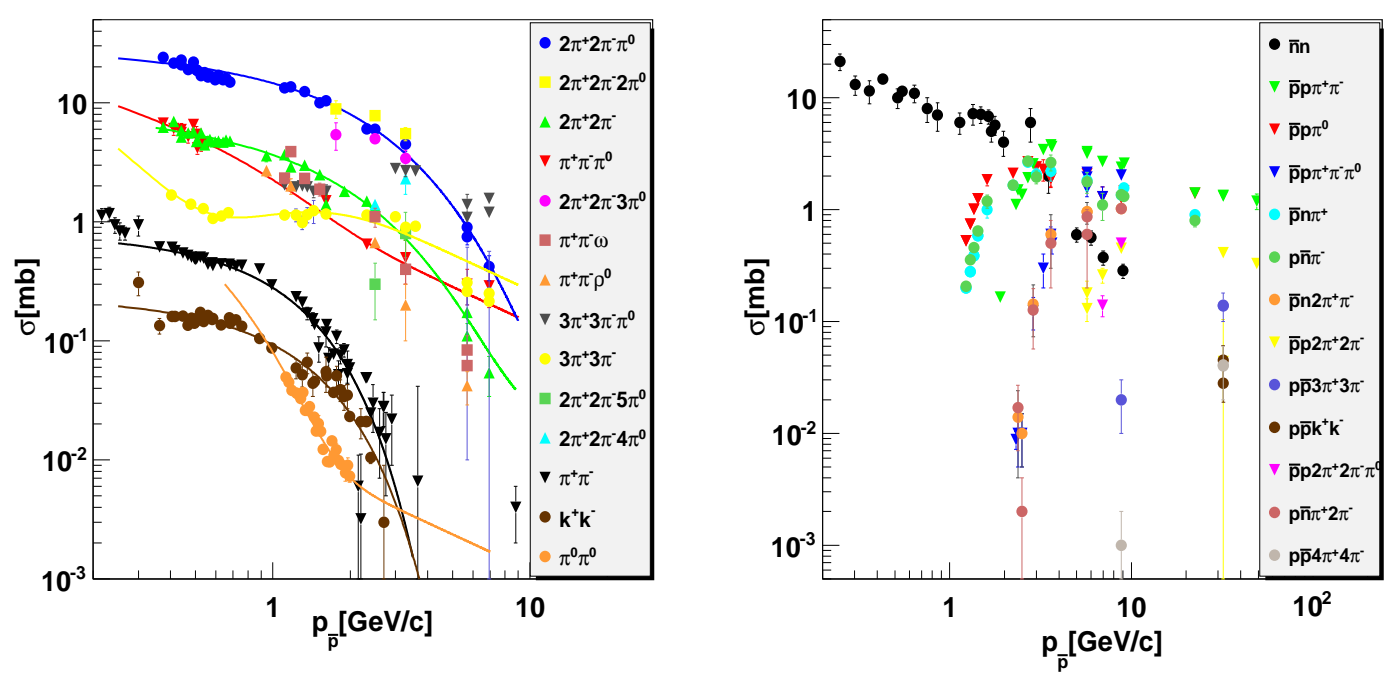

Figure 2: Cross sections for different inelastic channels in the $\bar{p}+p$ reaction as a function of the antiproton beam momentum $p_{\bar{p}}$ : (left) mesonic channels; (right) mesonic and hadronic channels

In this work we estimate the charge exhange (CE) contribution, using the formalism from Refs. $[11,12]$. The emission by the initial proton of a charged light meson- $\pi$ or $\rho$-meson in protonproton(anti-proton) collisions transforms high energy protons (for example in a proton beam) into neutrons. This effect is observed in accelerator physics [13]. Note that hard collinear photon emission was also studied in Ref. [14]. This works uses a frame where the relevant amplitude interferes with the background, i.e., all other emission mechanisms, except emission from the electron line, whereas [11] applies the structure function approach (that we use). Although these methods are different, the results obtained for the observables are equivalent.

CE reactions may occur in antiproton beams, too. At PANDA [15], FAIR [16], hard $\pi$ and $\rho$ meson can be detected with high efficiency. Charged meson will be deviated by the $2 \mathrm{~T}$ magnetic field of the central spectrometer, and (anti)neutrons, which are produced at high rate, could be used as a secondary high energy beam. We are interested here in 'initial state emission', as a mechanism to produce a (anti)neutron beam with comparable energy of the (anti)proton beam.

In this work, we propose a description of CE reactions referring to the known QED process of emission of a hard real photons by electron (positron) beams at $e^{+} e^{-}$colliders. Such process enhances the cross section when the energy loss from one of the incident particles lowers the total energy up to the mass of a resonance. This is known as "return to resonance" mechanism. In the case of creation of a narrow resonance this mechanism appears through a radiative tail: it is the characteristic behavior of the cross section which gradually decreases for energies exceeding the resonance mass. This mechanism provides, indeed, an effective method for studying narrow resonances like $J / \Psi$.

For the emission in a narrow cone along the directions of the initial (final) particles, the emission probability has a logarithmic enhancement, which increases with the energy of the "parent" charged particle. In frame of QED this mechanism is called as "quasi-real electron" mechanism 


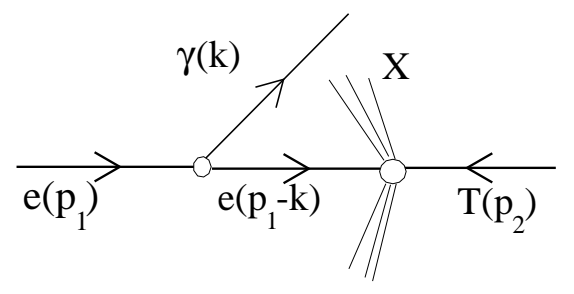

Figure 3: Feynman diagram for collinear hard photon emission in $e T$ reactions ( $\mathrm{T}$ stands for any target)

\section{(QRE) [11].}

In this work we apply the QRE mechanism to the case of hadrons and, in particular, to the collinear emission of a light meson from a (anti)proton beam. We evaluate the cross section for this process for single as well as multi pion production, where pions can be neutral or charged. Our derivation concerns a kinematical region outside the resonance formation region.

QRE mecanism is also known as Initial State Emission (ISR) method of scanning over incident energy, and can be used, in the hadron case, to produce different kind of particles in similar kinematical conditions. This can be very useful to measure the difference of the cross sections of the antiprotons and antineutrons scattering from the target. As a further step, it may open the way for checking sum rules with antiparticles (as Bjorken Sum Rule).

\section{Quasi-real electron method}

Let us consider the radiative process $e\left(p_{1}\right)+T\left(p_{2}\right) \rightarrow e\left(p_{1}-k\right)+\gamma(k)+X$ (the four momenta of the particles are written in parenthesis), $T$ stay for any nuclear target(proton $p$, or nucleus $A$ ), and the final state $X$ is undetected, Fig. 3 .

The virtual electron after the hard (collinear) photon emission is almost on mass shell [11]. This property allows to express the matrix element of the radiative process $e(p)+T \rightarrow e(p-k)+$ $\gamma(k)+X$ in terms of the matrix element of the non-radiative process $e+T \rightarrow e+X$ :

$$
\mathscr{M}_{\gamma}\left(p_{1}, p_{2}\right)=e \overline{\mathscr{T}}\left(p_{2}\right) \frac{\hat{p}_{1}-\hat{k}+m}{-2 p_{1} k} \hat{\varepsilon}(k) u\left(p_{1}\right) .
$$

The matrix element has a factorized form when the denominator of the intermediate electron's Green function is small: $\left|\left(p_{1}-k\right)^{2}-m^{2}\right| \ll 2 p_{1} p_{2}$. The matrix element squared, summed over the spin states of the photon is:

$$
\sum\left|\mathscr{M}_{\gamma}\right|^{2}=e^{2}\left[\frac{E_{p}^{2}+E_{\vec{p}-\vec{k}}^{2}}{\omega\left(E_{p}-\omega\right)(k p)}-\frac{m^{2}}{(k p)^{2}}\right] \sum\left|\overline{\mathscr{T}}\left(p_{2}\right) u\left(p_{1}-k\right)\right|^{2} .
$$

where $\sum\left|\overline{\mathscr{T}}\left(p_{2}\right) u\left(p_{1}-k\right)\right|^{2}$ is the Born matrix element squared with shifted argument.

In the case of unpolarized particles, the cross section of the process $e\left(p_{1}\right)+T\left(p_{2}\right) \rightarrow e+\gamma+T$ may be written in factorized form:

$$
\begin{aligned}
d \sigma_{\gamma}(s, x) & =d \sigma(\bar{x} s) d W_{\gamma}(x), \bar{x}=1-x, \\
d W_{\gamma}(x) & =\frac{\alpha}{\pi} \frac{d x}{x}\left[\left(1-x+\frac{1}{2} x^{2}\right) \ln \frac{E^{2} \theta_{0}^{2}}{m_{e}^{2}}-(1-x)\right], x=\frac{\omega}{E}, \theta<\theta_{0} \ll 1, \frac{E \theta_{0}}{m_{e}} \gg 1,
\end{aligned}
$$




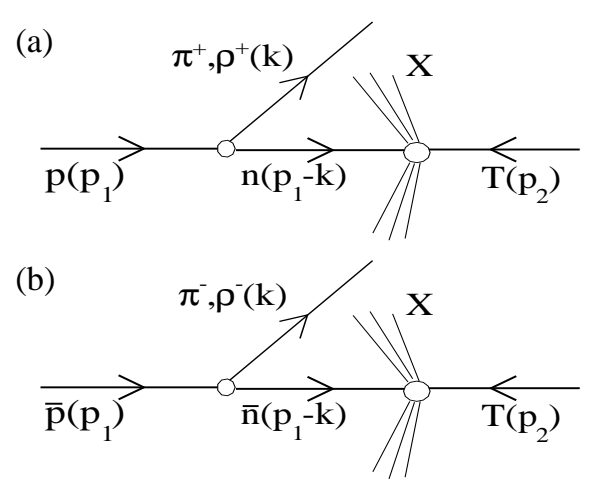

Figure 4: Feynman diagram for collinear hard charged pion ( $\rho$-meson) emission in $p(\bar{p})+T$ collisions.

where $E$ is the energy of the initial electron (center of mass frame implied $\vec{p}_{1}+\vec{p}_{2}=0$ ), and $s=\left(p_{1}+p_{2}\right)^{2}$.

It is assumed here that the initial electron transforms into an electron with energy fraction $1-x$ and a hard photon with energy fraction $x$ which is emitted within the cone $\theta<\theta_{0}$ along the direction of initial electron. Moreover it is implied that $\bar{x} s>s_{t h r}$, where $s_{t h r}$ is the threshold energy of process without photon emission. The logarithmic enhancement originates from the small values of the mass of the intermediate electron, which is almost on mass shell. This justifies the name of Quasi Real Electron (QRE) method. Such process has been also called Initial State Emission (ISR) method and allows scanning over the incident energy.

Below we consider a possible extension of the QRE method to the processes with "quasi real" (anti)nucleon intermediate state.

\section{Application to hadron physics}

Let us apply this formalism to the case of initial high energy proton (anti-proton) beams and the emission of a hard pion or vector meson in forward direction, collinear to the beam. We do not consider the emission of the pion from the final proton (final state emission), which corresponds to a different kinematical region for the intermediate particle.

For the case of emission of a positive-charged $\rho^{+}$or $\pi^{+}$meson by the high energy (anti)proton, the final state consists in a high energy (anti)neutron, accompanied by a positively charged meson. The charged meson can be deflected by an external magnetic field, providing the possibility to select a high energy neutron beam. In the case of emission of the neutral meson, it can be identified by measuring its decay channels.

Let us consider the reactions (Fig. 4):

$$
\begin{aligned}
& p+T \rightarrow n+T+h^{+} \\
& \bar{p}+T \rightarrow \bar{n}+T+h^{-}
\end{aligned}
$$

where $h=\rho$ or $\pi$ and $T$ may be any target ( $p, n$, nucleus..). In QED the interaction is assumed to occur through the exchange of a virtual photon. In the present case the particle can be a vector 
meson. However, the nature of the exchanged particle is irrelevant for the present considerations, where we focus on small angle charged pion emission and on the factorization properties of the cross section.

The matrix element for collinear $\pi(\rho)$ emission can be written as:

$$
\begin{aligned}
& \left.\mathscr{M}_{h_{+}}^{p T}\left(p_{1}, p_{2}\right)\right)=\mathscr{M}_{n T}\left(p_{1}-k, p_{2}\right) \mathscr{T}_{h_{+}}^{p n}\left(p_{1}, p_{1}-k\right), \\
& \left.\mathscr{M}_{h_{-}}^{\bar{p} T}\left(p_{1}, p_{2}\right)\right)=\mathscr{M}_{\bar{n} T}\left(p_{1}-k, p_{2}\right) \mathscr{T}_{h_{-}}^{\bar{p} \bar{n}}\left(p_{1}, p_{1}-k\right),
\end{aligned}
$$

where

$$
\begin{aligned}
& \mathscr{T}_{\pi}^{p n}\left(p_{1}, p_{1}-k\right)=\frac{g}{m_{h}^{2}-2 p_{1} k} \bar{u}_{n}\left(p_{1}-k\right) \gamma_{5} u_{p}\left(p_{1}\right), \\
& \mathscr{T}_{\rho}^{p n}\left(p_{1}, p_{1}-k\right)=\frac{g}{m_{h}^{2}-2 p_{1} k} \bar{u}_{n}\left(p_{1}-k\right) \hat{\varepsilon} u_{p}\left(p_{1}\right) .
\end{aligned}
$$

are the matrix elements of the subprocesses: $p \rightarrow n+\pi^{+}$and $p \rightarrow n+\rho^{+}$(or $\bar{p} \rightarrow \bar{n}+\pi^{-}$and $p \rightarrow n+\rho^{-}$). The matrix element of emission of a charged pion is expressed in factorized form (3.3) of a term connected to the target and a 'universal' factor for the hard meson emission, which generalizes the QED result. Below, we will focus on this last term.

The relevant cross sections are:

$$
\begin{aligned}
d \sigma^{p T \rightarrow h_{+} X}(s, x) & =\sigma^{n T \rightarrow X}(\bar{x} s) d W_{h_{+}}(x), \\
d \sigma^{\bar{p} T \rightarrow h_{+} X}(s, x) & =\sigma^{\bar{n} T \rightarrow X}(\bar{x} s) d W_{h_{-}}(x), \\
d \sigma^{p T \rightarrow h_{0} X}(s, x) & =\sigma^{p T \rightarrow X}(\bar{x} s) d W_{h_{0}}(x) .
\end{aligned}
$$

The quantity $d W_{\rho}(x)$ can be inferred using the QED result:

$$
\begin{aligned}
\frac{d W_{\rho^{i}}(x)}{d x} & =\frac{g^{2}}{4 \pi^{2}} \frac{1}{x} \sqrt{1-\frac{m_{\rho}^{2}}{x^{2} E^{2}}}\left[\left(1-x+\frac{1}{2} x^{2}\right) L-(1-x)\right], \\
1>x & =\frac{E_{\rho}}{E}>\frac{m_{\rho}}{E}, L=\ln \left(1+\frac{E^{2} \theta_{0}^{2}}{M^{2}}\right), \rho^{i}=\rho^{+}, \rho^{-}, \rho^{0}
\end{aligned}
$$

where $M, m_{\rho}, E$, and $E_{\rho}$ are the masses and the energies of the initial proton and the emitted $\rho$-meson (Laboratory reference frame implied).

For the probability of hard pion emission we have

$$
\frac{d W_{\pi}}{d x}=\sum\left|\mathscr{T}_{p n}\left(p_{1}, p_{1}-k\right)\right|^{2} \frac{d^{3} k}{16 \omega \pi^{3}},
$$

with

$$
\begin{array}{r}
\sum\left|\mathscr{T}^{p n}\left(p_{1}, p_{1}-k\right)\right|^{2}=\frac{g^{2}}{\left[m_{\pi}^{2}-2\left(p_{1} k\right)\right]^{2}} \operatorname{Tr}\left(\hat{p}_{1}-\hat{k}+M\right) \gamma_{5}\left(\hat{p}_{1}+M\right) \gamma_{5}= \\
\frac{4\left(p_{1} k\right) g^{2}}{\left[m_{\pi}^{2}-2\left(p_{1} k\right)\right]^{2}},\left(p_{1} k\right)=E \omega(1-b c), 1-b^{2} \approx \frac{m_{\pi}^{2}}{\omega^{2}}+\frac{M^{2}}{E^{2}}
\end{array}
$$




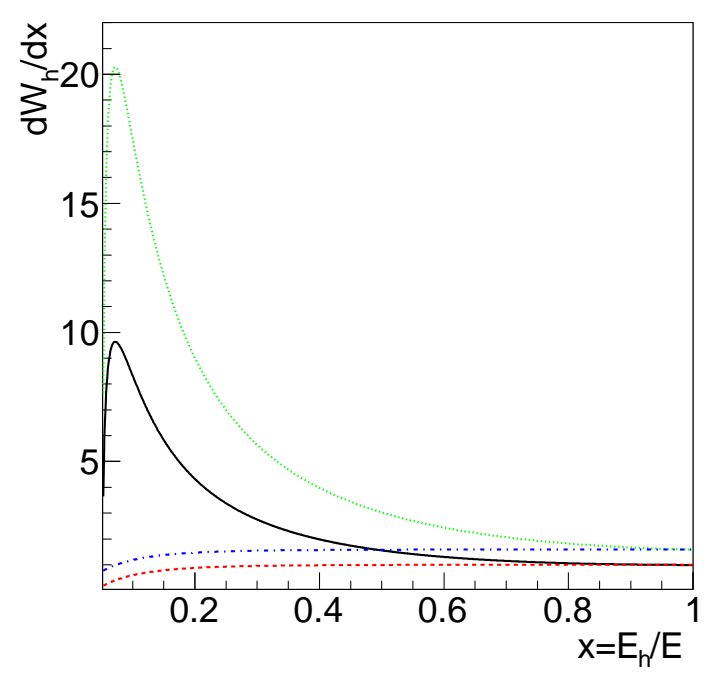

Figure 5: Color online. Quantity $d W_{h} / d x$ for $\rho$ - and $\pi$-meson production as a function of the meson energy fraction $x=E_{h} / E$, from Eqs. (3.6,3.9), for $E=15 \mathrm{GeV}$ and for two values of the $h$-emission angle: $\theta_{0}=10^{\circ}$ for $\rho$ - meson (black, solid) and for $\pi$-meson (red, dashed), $\theta_{0}=20^{\circ}$ for $\rho$ - meson (green, dotted) and for $\pi$-meson (blue, dash-dotted).

with $c=\cos \left(\vec{k}, \vec{p}_{1}\right)$. The angular integration in the region $1-\left(\theta_{0}^{2} / 2\right)<c<1$ leads to

$$
\begin{aligned}
& \frac{d W_{\pi^{i}}(x)}{d x}=\frac{g^{2}}{8 \pi^{2}} \sqrt{1-\frac{m_{\pi}^{2}}{x^{2} E^{2}}}\left[L+\ln \frac{1}{d(x)}+\frac{m_{\pi}^{2}}{x d(x) M^{2}}\right], \\
& 1>x=\frac{E_{\pi}}{E}>\frac{m_{\pi}}{E}, d(x)=1+\frac{m_{\pi}^{2} \bar{x}}{M^{2} x^{2}}, \bar{x}=1-x, \pi^{i}=\pi^{+}, \pi^{-}, \pi^{0},
\end{aligned}
$$

where $g=g_{\rho p p}=g_{\pi p p} \approx 6$ is the strong coupling constant. The quantities $d W_{h}(x) / d x$ as functions of the energy fraction $x=E_{h} / E(h=\rho, \pi)$ are drawn in Fig. 5, for $E=15 \mathrm{GeV}$ and two different values of $\theta_{0}: 10^{\circ}$ and $20^{\circ}$.

The expressions of the integrated probabilities are:

$$
\begin{aligned}
& W_{h}=\int_{x_{t}^{h}}^{1} \frac{d W_{h}}{d x} d x=\frac{g^{2}}{4 \pi^{2}}\left(A^{h} L+B^{h}\right), \\
& A^{\rho}=I_{0}\left(x_{t}^{\rho}\right)-I_{1}\left(x_{t}^{\rho}\right)+\frac{1}{2} I_{2}\left(x_{t}^{\rho}\right), B^{\rho}=-I_{0}\left(x_{t}^{\rho}\right)+I_{1}\left(x_{t}^{\rho}\right), \\
& A^{\pi}=\frac{1}{2} I_{1}\left(x_{t}^{\pi}\right) ; B^{\pi}=I_{1}\left(x_{t}^{\pi}\right),
\end{aligned}
$$

where $x_{t}^{h}=E_{t}^{h} / E$ with $E_{t}^{h}=m^{h}$ - threshold value of the energy of the detected particle, $h=\rho, \pi$. The analytic expressions of the integrals

$$
I_{n}(z)=\int_{z}^{1} \frac{d x}{x} x^{n} \sqrt{1-\left(\frac{z}{x}\right)^{2}}
$$


are:

$$
\begin{aligned}
& I_{0}(z)=\frac{1}{2} \ln \frac{1+r}{1-r}-r \\
& I_{1}(z)=r+z \arcsin (z) \\
& I_{2}(z)=\frac{1}{2} r-\frac{z^{2}}{4} \ln \frac{1+r}{1-r}
\end{aligned}
$$

with $r=\sqrt{1-z^{2}}$.

The integrated quantities $W_{h}, h=\rho, \pi$ can, in general, exceed unity, violating unitarity. To restore unitarity, we have to take into account virtual corrections: the vertex for the emission of a single pion (charged or neutral) from the proton has to include 'radiative corrections', which account for emission and absorption of any number of virtual pions. For this aim we use the known expression for the probability of emission of $n$ "soft" photons in processes of charged particles hard interaction, i.e., the Poisson formula for emission of $n$ soft photons $W^{n}=\left(a^{n} / n !\right) e^{-a}$ (where $a$ is the probability of emission of a single soft photon) [17].

Note that the probability of emission of 'soft' neutral pions follows a Poisson distribution, which is not the case for the emission of charged pions. Fortunately, in our case, it is sufficient to consider the emission of one charged pion at lowest order (the process of one charged pion emission) plus any number of real and virtual pion with total charge zero. In such configuration, this vertex has the form of the product of the Born probability of emission of a single pion multiplied by the Poisson-like factor:

$$
P_{\pi, \rho}=e^{-W_{\pi, \rho}},
$$

which takes into account virtual corrections.

The renormalized probabilities $W_{\pi, \rho} P_{\pi, \rho}<1$ from Eqs. $(3.10,3.11)$ are illustrated in Fig. 6 as a function of energy, for two different $\theta_{0}$ angles.

Keeping in mind the possible processes of emission of $n$ real soft neutral pion escaping the detection, the final result can be obtained using the replacement

$$
\sigma(s) \rightarrow \sigma(s) \times \mathscr{R}_{\pi}, \mathscr{R}_{\pi}=P_{\pi} \sum_{k=0}^{k=n} \frac{W_{\pi}^{k}}{k !} .
$$

The renormalization factor $\mathscr{R}_{\pi}$ is illustrated in Fig. 7, for the probability of emission of 2 (black, solid line), 3 (red, dashed line), 4 (green, dotted line) pions.

The quantity $P_{\pi}$ can be compared to the experimentally measurable phenomena [13]: the fraction of protons in the final state of proton-proton collisions is approximately one half, $P_{\pi} \approx 0.5$. The commonly accepted explanation is that charge exchange reactions are responsible for changing protons into neutrons.

Concerning the production of two charged pions, accompanied by a final state $X$, we can write:

$$
d \sigma^{p \bar{p} \rightarrow \rho^{0} X}=2 \frac{d W_{\rho}(x)}{d x} \sigma^{p \bar{p} \rightarrow X}(\bar{x} s) \times P_{\rho},
$$

where the factor of two takes into account two kinematical situations, corresponding to the emission along each of the initial particles and $P_{\rho}$ is the survival factor (3.11) which takes into account virtual radiative corrections. The characteristic peak at $x=x_{\max }$ has the same nature as for the QED process 


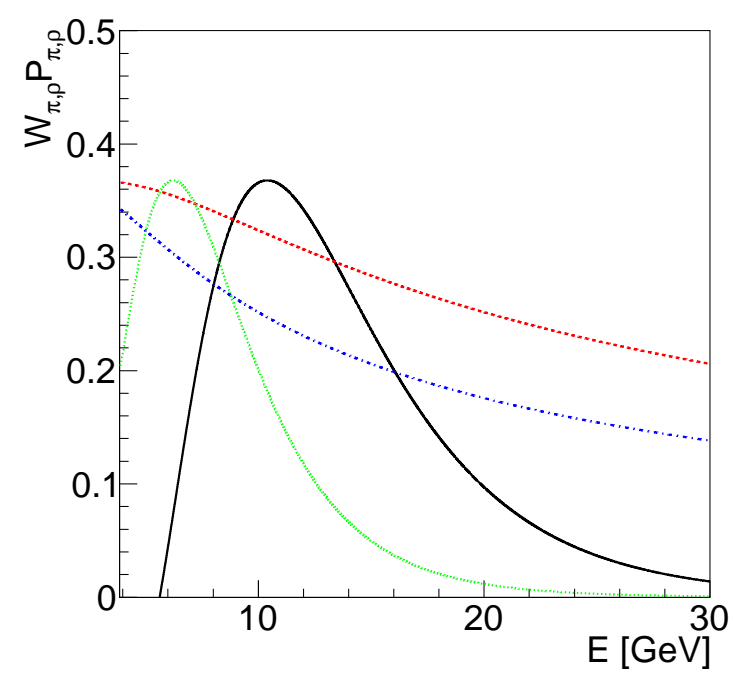

Figure 6: Renormalized probabilities $W_{\rho, \pi} \cdot P_{\rho, \pi}$, as function of the incident energy for two values of the hadron emission angle.

$e^{+}+e^{-} \rightarrow \mu^{+}+\mu^{-}+\gamma$. As explained in Ref. [18], it is a threshold effect, corresponding to the creation of a muon pair, where $x_{\max }=1-4 M_{\mu}^{2} / s, M_{\mu}$ is the muon mass. Let us consider the antiproton-proton annihilation into two and three pions $\bar{p}+p \rightarrow \pi^{+} \pi^{-}$or $\bar{p}+p \rightarrow \pi^{+} \pi^{-} \pi^{0}$.

The cross section (3.13) is illustrated in Fig. 8 for two different values of the laboratory energy and of the emitted angle as a function of the $\rho$ meson energy fraction.

In case of three pion production, assuming that the process occurs through a $\pi^{0} \rho^{0}$ initial state emission, we find:

$$
\begin{aligned}
d \sigma(p, \bar{p})^{p \bar{p} \rightarrow \pi \rho X}= & d W_{\rho}^{0}\left(x_{\rho}\right) d W_{\pi}^{0}\left(x_{\pi}\right)\left[d \sigma\left(p-p_{\rho}, \bar{p}-p_{\pi}\right)^{p \bar{p} \rightarrow X}+\right. \\
& \left.d \sigma\left(p-p_{\pi}, \bar{p}-p_{\rho}\right)^{p \bar{p} \rightarrow X}\right] P_{\pi} P_{\rho},
\end{aligned}
$$

implying the subsequent decay $\rho^{0} \rightarrow \pi^{+} \pi^{-}$.

It is interesting to note that the cross sections for the interaction of high energy neutron (antineutron) beams with a hadronic target can be calculated using the cross sections of proton beam interacting with the same target with the emission of the charged meson. We obtain (see Eqs. $(3.6,3.9))$ :

$$
\sigma^{n T \rightarrow X}(\bar{x} s)=\frac{d \sigma^{p T \rightarrow h^{+} X} / d x}{d W_{+}(x) / d x}
$$

and similarly for the anti-proton beams.

Experimental data from coincidence experiment with the selection of a hard meson at small angles are not available, and we can not test the factorisation prediction. However the values of the cross section for $\bar{p} p \rightarrow \rho^{0} X$ illustrated in Fig. 8 are not in contradiction with the corresponding total cross sections (for a compilation, see [2]). 


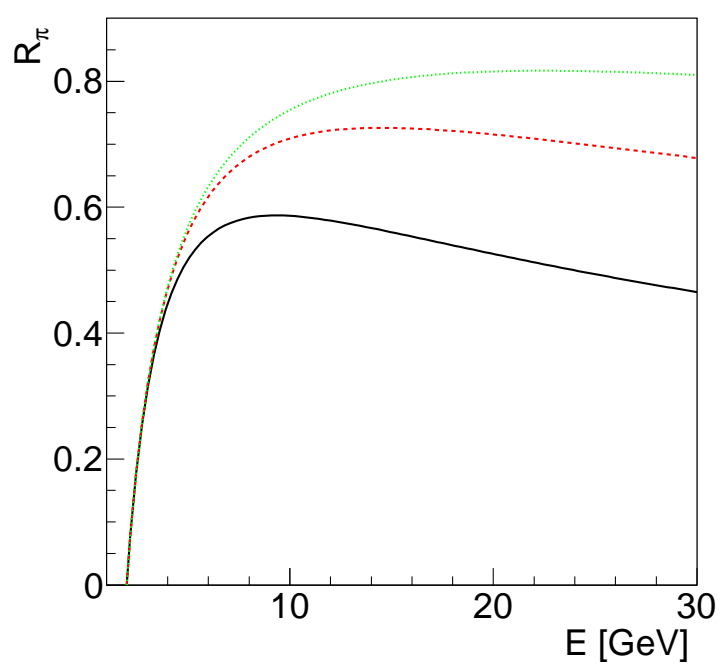

Figure 7: Renormalization factor $R_{\pi}$, for the probability of emission of 2 (black, solid line), 3 (red, dashed line), 4 (green, dotted line) neutral pions, as a function of the incident energy for $\theta_{0}=10^{\circ}$.

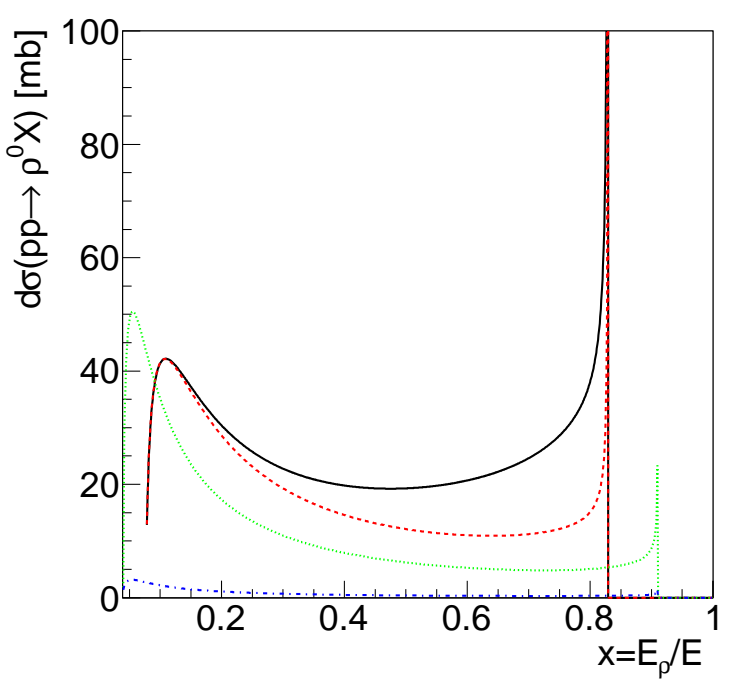

Figure 8: The cross section $d \sigma\left(p \bar{p} \rightarrow \rho^{0} X\right)$ is plotted as function of the $\rho$ energy fraction for two values of the incident energy and of the $\rho$ emission angle: $E=10 \mathrm{GeV}$ and $\theta_{0}=10^{\circ}$ (black, solid line), $E=10 \mathrm{GeV}$ and $\theta_{0}=20^{\circ}$ (red, dashed line), $E=20 \mathrm{GeV}$ and $\theta_{0}=10^{\circ}$ (green, dotted line), $E=20 \mathrm{GeV}$ and $\theta_{0}=20^{\circ}$ (blue, dash-dotted line). 
Let us discuss the limits of our approach and the accuracy of the present calculation. Concerning the kinematical region where this mechanism is important, we consider charged pion emission at angles, smaller than an angle $\theta_{0}$, i.e. $\theta<\theta_{0} \ll 1$. Moreover $E \theta>>M$, which insures the presence of large logarithm. In this case, the main contribution is due to the large quantity $L=\ln \left(E^{2} \theta^{2} / M^{2}\right) \gg 1$. In the calculations, we keep the terms containing $L$ and omit the terms proportional to $\theta^{2}$. The resulting accuracy is given by $1+O(1 / L)$. Taking $L=10$, the accuracy of the calculation is of the order of $10 \%$.

We considered here only initial proton emission. In this case, the resonance formation is kinematically forbidden since the four momentum of the virtual nucleon is Space-Like. The simplest CE processes $p \bar{p} \rightarrow n \bar{n}, \bar{p} n \rightarrow \bar{\Lambda} \Sigma^{-}$can be in principle measured at PANDA. Other reaction mechanisms can contribute to these processes. In the frame of a description in terms of a single pseudoscalar meson $\pi^{+}, K^{+}$exchange, information on the strange meson-baryon constant can be extracted. Note that neutrons created by $\rho$ and $\pi$ emission form a (anti)proton beam with the mechanism considered here (initial state radiation) can not be formed by subsequent decay of a nucleon resonance, as $\Delta$. Nucleon resonances can not be produced in the initial state, because the neutron has the time like momentum squared (the propagator is $\left.(p-k)^{2}-M^{2}<0\right)$.

\section{Instead of Conclusions: A memory of Professor Eduard Alekseevich KURAEV}

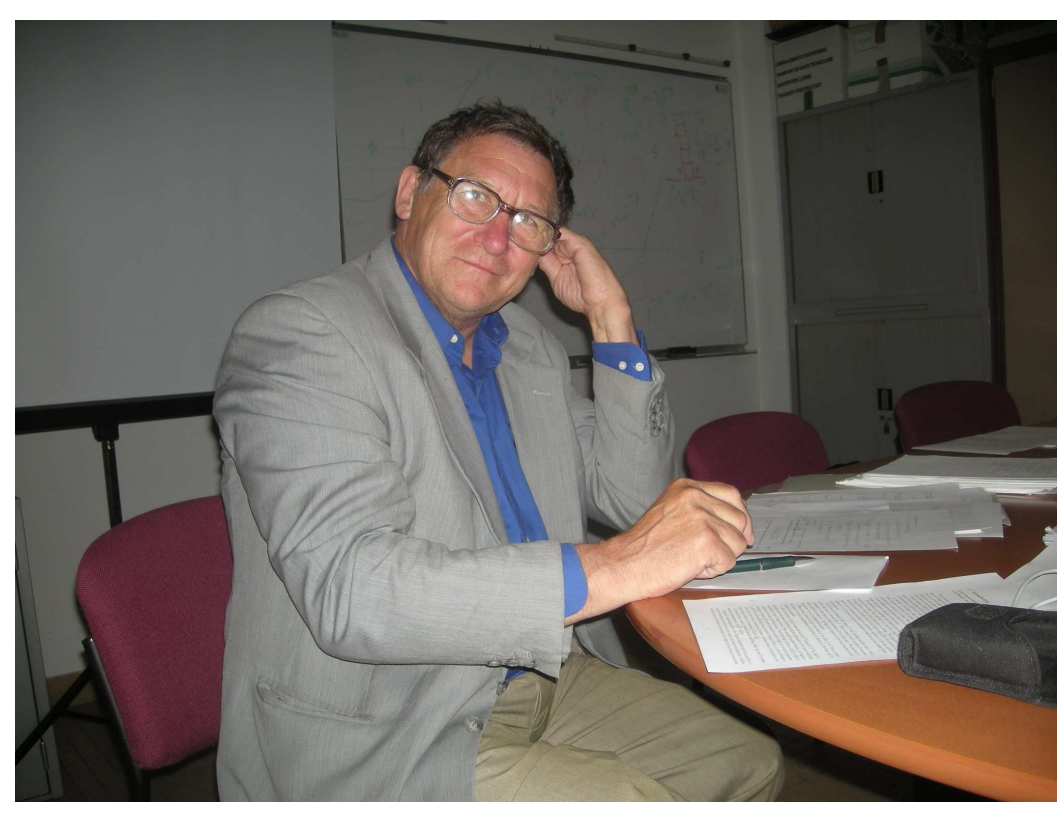

Prof. Eduard Alekseevich KURAEV

Krasnodar, 17/10/1940 Dubna, 4/3/2014

Prof. Eduard Alekseevich KURAEV passed away, while working on this subject.

It is an invaluable loss for our community, in particular for the colleagues who had the chance to meet him and to share his encyclopedical knowledge, his enthusiasm, and his original and surprising ideas. 
Always concentrated on solving difficult problems, He published hundreds of scientific papers, and collected thousands of citations.

He had an astonishing energy, extraordinary ability and resistance for effort and hard work, a passion for sharing ideas, discoveries, and results in seminars and in teaching and educating young students and researchers. He was always ready to listen and discuss with the colleagues knocking at his door, coming from near or far Institutes.

He had a deep understanding and a curiosity in the observation of Nature, of the visible and invisible features of Nature, and, at the same time, a strong capability in abstraction and a passion for mathematical problems.

We loose with Prof. Eduard Alekseevich Kuraev, an engaging and unique personality, and an exceptional physicist.

\section{References}

[1] V. N. Baier, V. S. Fadin and V. A. Khoze, Nucl. Phys. B 65, 381 (1973).

[2] A. Dbeyssi and E. Tomasi-Gustafsson, Prob.Atomic Sci.Technol. 2012N1, 84 (2012); PANDA internal note AD12-12-13

[3] E. A. Kuraev, E. S. Kokoulina and E. Tomasi-Gustafsson, arXiv:1306.5169 [hep-ph].

[4] V. S. Murzin and L, I. Sarycheva. Multiparticle processes at high energies. Atomizdat, M. (in Russian) 1974, p. 253, and references therein; S. Baskovic et al. Yad. Phys., 27, 1225 (1978).

[5] V.V. Avdeichikov et al. Instrum. Exp. Tech. 56 (2013) 9.

[6] M. A. Braun, Y. .A. Evlashev and V. VVechernin, Yad. Fiz. 27 (1978) 1329.

[7] E. A. Kuraev, S. Bakmaev and E. A. Kokoulina, Nucl. Phys. B 851 (2011) 551.

[8] E. N. Antonov, L. N. Lipatov, E. A. Kuraev and I. O. Cherednikov, Nucl. Phys. B 721 (2005) 111.

[9] L. N. Lipatov, Nucl. Phys. B 365 (1991) 614.

[10] S. Gasiorowicz, Elementary particle physics, J. Wiley \& Sons Inc., New York (1966).

[11] V. Baier, V. Fadin and V. Khoze, Nucl. Phys. B65, 381 (1973).

[12] A. B. Arbuzov, V. V. Bytev, E. A. Kuraev, E. Tomasi-Gustafsson and Y. .M. Bystritskiy, Phys. Part. Nucl. 41, 593 (2010); Phys. Part. Nucl. 42, 1 (2011).

[13] V. A. Nikitin, private communication.

[14] M. Caffo, R. Gatto and E. Remiddi, Nucl. Phys. B 252 (1985) 378.

[15] Physics Performance Report for PANDA: Strong Interaction Studies with Antiprotons, The PANDA Collaboration, arXiv:0903.3905 [hep-ex]; http://www.gsi.de/PANDA;

[16] http://www.gsi.de/FAIR;

[17] A. I. Akhiezer and V. B. Berestetskij, Quantum Electrodynamics, Moscow Science, 1981.

[18] V.N. Baier, V.S. Fadin and V.M. Katkov, Emission of Relativistic Electrons, Atomizdat, Moscow, 1973 (in Russian).

[19] E.S. Kokoulina, Acta Physica Polonica. B35, 295 (2004); M.V. Volkov and E.S Kokoulina, Phys.Part. Nucl. Lett. 1, 16 (2004). 